\title{
CONSIDERACIONES SOBRE LA COMUNIDAD DE DIATOMEAS EN RELACIÓN A GRADIENTES DE ALTITUD Y SALINIDAD EN humedales de la Puna y los Altos Andes (Catamarca Y JuJuY, ARgentinA)
}

\section{CONSIDERATIONS ON THE COMMUNITY OF DIATOMS IN RELATION TO GRADIENTS of Altitude and Salinity in wetlands of Puna and Andes of High ALTITUdE FROM CATAMARCA AND JUJuY, ARgENTINA}

\author{
Claudia T. Seeligmann ${ }^{1}$ (iD y Nora I. Maidana ${ }^{2,3}$ (1)
}

1. Universidad Nacional de Tucumán, Facultad de Ciencias Naturales e IML, Argentina.

2. Universidad de Buenos Aires, Facultad de Ciencias Exactas y Naturales, Departamento de Biodiversidad y Biología Experimental, Laboratorio de Diatomeas Continentales, Buenos Aires, Argentina.

3. CONICET- Universidad de Buenos Aires, Instituto de Biodiversidad y Biología Experimental y Aplicada (IBBEA), Laboratorio de Diatomeas Continentales, Buenos Aires, Argentina.

*claudiaseeligmann@gmail.com

Citar este artículo SEELIGMANN, C. T. \& N. I. MAIDANA. 2019. Consideraciones sobre la comunidad de diatomeas en relación a gradientes de altitud y salinidad en humedales de la Puna y los Altos Andes (Catamarca y Jujuy, Argentina). Bol. Soc. Argent. Bot. 54: 475-486.

DOI: http://dx.doi. org/10.31055/1851.2372.v54. n4.23902

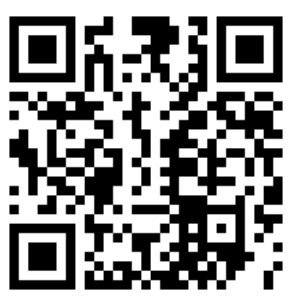

Recibido: 4 Abril 2019

Aceptado: 25 Octubre 2019

Publicado: 15 Diciembre 2019

Editora: Luz Allende iD

\section{SUMmary}

Background and Aims: In the Argentinean Puna and Prepuna there is an important number of high altitude wetlands from purely fresh to frankly hyperhaline. This contribution aimed to integrate the information collected in eight years of study (1998 and 2005) and analyze the distribution of taxa along gradients of height (2600-4700 $\mathrm{m}$ asl) and salinity $\left(0.2-104 \mathrm{~g} \mathrm{~L}^{-1}\right)$.

M\&M: We analyzed the diatoms found in our previous surveys of 57 wetlands located in the provinces of Catamarca and Jujuy between 2340 and $4683 \mathrm{~m}$ asl.

Results: We identified 73 genera of diatoms with 375 infrageneric taxa and many of them recorded for the first time in the country (29) or in the region of our study (331). The best genera represented in terms of number of species were Nitzschia (43), Navicula (37), Pinnularia (21), Surirella (17), Planothidium (15), Halamphora (13) Craticula (12), Amphora and Stauroneis 10). In the oligohaline wetlands, specific richness fluctuated between 7 and 69, mesohaline between 18 and 54 and polyhaline between 29 and 49. Regarding the height, between 4,301-4,500 m asl, there was a significant decrease in the number of species.

Conclusions: The species richness does not seem to have a direct relation with the altitude and salinity, reason why we postulate that other factors have influence on the diversity in the environments of high altitude analyzed. In this contribution, we report new ranges of tolerance to salinity and height for many taxa.

\section{KEY WORDS}

Bacillariophyceae, salinity, altitude, wetlands.

\section{RESUMEN}

Introducción y objetivos: En la Puna y Prepuna Argentina existe un importante número de humedales de altura netamente dulces hasta francamente hiperhalinos. Este aporte tuvo como objetivos integrar la información recabada en ocho años de estudio (1998 y 2005) y analizar la distribución de los taxones a lo largo de gradientes de altura (2600-4700 m snm) y salinidad (0,2-104 g L-1).

M\&M: Analizamos las diatomeas halladas en 57 humedales ubicados en las provincias de Catamarca y Jujuy entre los 2340 y $4683 \mathrm{~m} \mathrm{snm}$ de altura.

Resultados: Identificamos 73 géneros de diatomeas con 375 taxones infragenéricos y a muchos de ellos los registramos por primera vez en el país (29) o en la región de estudio (331). Los géneros mejor representados en cuanto al número de especies fueron: Nitzschia (43), Navicula (37), Pinnularia (21), Surirella (17), Planothidium (15), Halamphora (13) Craticula (12), Amphora (11) y Stauroneis (10). En los humedales oligohalinos, la riqueza específica fluctuó entre 7 y 69, en los mesohalinos entre 18 y 54 y en los polihalinos entre 29 a 49. Respecto a la altura, entre los 4301-4500 m snm, ocurrió una importante disminución del número de especies.

Conclusiones: La riqueza específica no parece tener una relación directa con la altitud o la salinidad por lo que postulamos que serían otros factores los que condicionarían la diversidad en los ambientes de altura analizados. En este aporte damos a conocer nuevos rangos de tolerancia a la salinidad y la altura para numerosos taxones.

Palabras Clave

Bacillariophyceae, salinidad, altitud, humedales. 


\section{INTRODUCCIÓN}

Los humedales de alta montaña son particularmente sensibles a los cambios climáticos y a los efectos antrópicos debido a su baja resiliencia. Se encuentran en geografías con drenajes esporádicos, arreicos y condiciones climáticas extremas, por lo que reaccionan rápidamente al estrés ambiental.

En Argentina, los ambientes de alta montaña se ubican en la Subregión Páramo Puneña de la Región Andina (Morrone, 2006). Este autor considera cinco provincias dentro de la subregión Páramo Puneña, de las cuales la Puna y Prepuna incluyen los ambientes de alta montaña de Argentina. Estas provincias están sometidas a grandes amplitudes térmicas, fuerte irradiación solar con precipitaciones que, en forma de lluvias, nieve y/o granizo, no superan los $200 \mathrm{~mm}$ anuales. Con estas condiciones, los humedales de esta región registran fluctuaciones notables del espejo y nivel del agua y, en su mayoría, tienden a ser salinos debido al aporte del entorno geológico y a la relación que se establece entre la baja precipitación y la alta evaporación. Entre la gran diversidad de los humedales de altura, se pueden hallar lagunas hiperhalinas $\left(>60.000 \mathrm{scm}^{-1}\right)$ y muy someras $(<$ $1 \mathrm{~m})$ y otras con mayor profundidad y dulces o mesohalinas (Caziani et al., 2001).

La mayor parte de los cuerpos de agua de alta montaña han sido objeto de escasa atención por parte de los limnólogos, quedando indefensos frente a la creciente incidencia antrópica. El escaso conocimiento de estos ambientes podría deberse, en parte, a su estratégica ubicación y difícil acceso (Williams, 1993; Caziani \& Derlindati, 2000). A pesar de la alta diversidad de los humedales de la Puna y Prepuna (Cazianni et al., 2001) solo muy pocos fueron abordados en relación a determinar el status de conservación. En tal sentido, Laguna Pozuelos fue ampliamente estudiada ecológicamente, conocimiento que llevó a declararla Sitio Ramsar en 1992 (García Fernández \& Tecchi, 1991; Mascitti, 1996; Salusso et al., 1997; Maidana et al., 1998; González Achem et al., 2014).

El cordón montañoso andino, con sus grandes extensiones, variada topografía y diferentes condiciones climáticas crea un escenario ideal para el desarrollo de una flora diatomológica heterogénea (Rumrich et al., 2000). Es por ello que en las últimas décadas se ha incrementado el interés por conocer la diatomoflora de humedales de altura y entre los aportes sobre diatomeas de los Andes se pueden citar las contribuciones realizadas por Rumrich et al. (2000); ÁlvarezBlanco et al. (2011); Blanco et al. (2013); Díaz \& Maidana (2005); González Achem et al. (2014); Maidana \& Seeligmann (2006, 2015); Maidana et al. (2009 y 2011); Morales et al. (2012); Seeligmann \& Maidana (2003, 2013); Seeligmann et al. (2008), entre otros. Estos estudios, en su mayoría, se centraron en la taxonomía, quedando como incógnita la información relacionada con los factores que regulan la riqueza y abundancia de las comunidades planctónicas y bentónicas. Rejas et al. (2012) y Frau et al. (2015) propusieron, de acuerdo a sus registros, que la disponibilidad de nutrientes (principalmente nitrógeno y fósforo), salinidad y altitud eran los factores que controlaban principalmente la composición y abundancia de las algas. En ambientes de altura, la riqueza específica de las diatomeas no siempre mostró una relación clara con la salinidad (Blanco et al., 2013; Frau et al., 2015; Maidana et al., 2009). Servant-Vildary \& Blanco (1984) consideran a la química del agua como la restricción limnológica principal que modela la estructura de las comunidades algales. En humedales salinos de altura Frau et al. (2015) hallaron en Catamarca que en los lagos hipohalinos (3-20 $\left.\mathrm{gL}^{-1}\right)$ y mesohalinos $\left(20-50 \mathrm{gL}^{-1}\right)$, según la clasificación dada por Hammer (1986), las muestras tuvieron como dominantes a las Bacillariophyceae, con los géneros Surirella Turpin y Navicula Bory.

Las elevadas salinidades de estos ambientes representan una primera barrera para el desarrollo de los organismos que deben adaptarse, por ejemplo, a alta presión osmótica, densidad óptica y baja solubilidad del oxígeno, variables que tienen una importante influencia sobre los organismos fotosintéticos (Bucher, 2006).

En un estudio realizado en lagunas de la Puna jujeña, Salusso et al. (1997) observaron que las diferencias entre los humedales estuvieron, principalmente, dadas por las concentraciones de iones, las que a su vez estaban fuertemente condicionadas por la geología del entorno.

En esta contribución se dan a conocer los resultados obtenidos de estudios diatomológicos realizados en 57 cuerpos de agua (ríos, vegas y lagunas), en las provincias argentinas de Catamarca 


\section{T. Seeligmann y N. I. Maidana - Altitud y salinidad vs. diatomeas de gran altitud}

y Jujuy. Los objetivos propuestos fueron integrar la información recabada en los ocho años de estudio y analizar la distribución de los taxones a lo largo de gradientes amplios de altura (2600-4700 m snm) y salinidad $\left(0,2-104 \mathrm{~g} \mathrm{~L}^{-1}\right)$.

\section{Materiales y Métodos}

Se analizaron los resultados de las investigaciones realizadas sobre las diatomeas presentes en muestras de agua de 57 humedales de altura obtenidas durante un período de 8 años (1998 y 2005), en ríos, vegas y lagunas ubicadas en las provincias de Catamarca (35) y Jujuy (22) entre los 2340 y 4683 $\mathrm{m}$ snm y entre $22^{\circ}-28^{\circ} \mathrm{S}$ y $65^{\circ}-69^{\circ} \mathrm{O}$ (Fig. 1). En cada humedal se tomaron 3 muestras de agua, tratando de abarcar, en lo posible la heterogeneidad del ambiente. Se midieron in situ temperatura, $\mathrm{pH}$, conductividad y, en algunos casos, se contó con datos (inéditos) de análisis químicos propios y otros proporcionados por el Grupo de Conservación de Flamencos Altoandinos (GCFA). La salinidad fue estimada según la fórmula de Dejoux (1993). Para clasificar los humedales de acuerdo a su salinidad, se utilizó el sistema de Cowardin et al. (1979) para aguas continentales: dulces $\left(<0,5 \mathrm{~g} \mathrm{~L}^{-1}\right)$, oligohalinos 0,5-5 $\left(\mathrm{g} \mathrm{L}^{-1}\right)$, mesohalinos $\left(5-18 \mathrm{~g} \mathrm{~L}^{-1}\right)$, polihalinos (18-30 $\left.\mathrm{g} \mathrm{L}^{-1}\right)$, hiperhalinos $\left(>40 \mathrm{~g} \mathrm{~L}^{-1}\right)$.

Los requerimientos ecológicos de las distintas especies fueron consultados en Lowe (1974), De Wolf (1982), Van Dam et al. (1994), y de la literatura consultada en cada caso.

\section{Resultados}

Desde el punto de vista químico, la mayoría de los humedales registraron aguas desde neutras a alcalinas ( $\mathrm{pH}$ 7-11). En relación a la salinidad, los ríos, vegas y algunas lagunas, la mayor parte de ellas ubicadas en Jujuy, se incluyeron dentro del tipo de aguas netamente dulces. La mayoría de los humedales hiperhalinos estudiados están en Catamarca y, entre ellos, se destacaron por sus altos registros de salinidad, las lagunas Diamante $(73,9 \mathrm{~g}$ $\left.\mathrm{L}^{-1}\right)$ (Fig. 1B; sitio 28), Carachi Pampa $\left(68,8 \mathrm{~g} \mathrm{~L}^{-1}\right.$; Fig. 1B; sitio 14), Caro (68,4 $\mathrm{g} \mathrm{L}^{-1}$; Fig. 1B; sitio 29), Cabi (51,8 g L L$^{-1}$; Fig. 1B; sitio 18), Purulla (50,3 $\mathrm{g} \mathrm{L}^{-1}$; Fig. 1B; sitio 13) y La Lagunita (50 g
$\mathrm{L}^{-1}$; Fig. 1B; sitio 12). En Jujuy, sobresalieron por su grado salino las lagunas Guinda $\left(105 \mathrm{~g} \mathrm{~L}^{-1}\right.$; Fig. 1A; sitio 10) y Honda (99 $\mathrm{g} \mathrm{L}^{-1}$; Fig. 1A; sitio 8). La salinidad de cada cuerpo de agua no tuvo una relación directa con la altitud a la que se encuentra (Fig. 2).

En relación a los iones mayoritarios, predominaron las aguas de tipo sódico-cloradas, con una secuencia $\mathrm{Cl}^{-}>\mathrm{SO}_{4}>>\mathrm{HCO}_{3}^{-}$. Los valores más altos de $\mathrm{Na}^{+}$y cloruros se obtuvieron en la

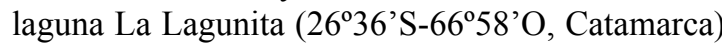
con $113 \mathrm{~g} \mathrm{~L}^{-1} \mathrm{de} \mathrm{Na}^{+}$y $235 \mathrm{~g} \mathrm{~L}^{-1}$ de cloruros. En otras lagunas de la misma provincia, como Baya (Fig. 1B; sitio 20), Carachi Pampa, Caro, Diamante, Grande (Fig. 1B; sitio 19) y Purulla, las aguas también fueron altamente cloruradas, con lecturas de más de $58 \mathrm{~g} \mathrm{~L}^{-1}$. No obstante, Carachi Pampa, Diamante y Baya fueron además marcadamente sulfatadas, con registros de hasta $35 \mathrm{~g} \mathrm{~L}^{-1}$, aunque los cloruros se encontraron como dominantes. También en Jujuy, las lagunas Runtuyoc (Fig. 1A; sitio 3) y Catal (Fig. 1A; sitio 21) tuvieron mayor cantidad de sulfatos que de cloruros, siendo oligohalina la primera, con $0,15 \mathrm{~g} \mathrm{~L}^{-1}$ de sulfatos y $0,045 \mathrm{~g} \mathrm{~L}^{-1}$ de cloruros y polihalina la segunda, con $7 \mathrm{~g} \mathrm{~L}^{-1}$ de sulfatos y 2,7 $\mathrm{g} \mathrm{L}^{-1}$ de cloruros. Cabe destacar que en algunas lagunas, como Purulla en Catamarca, se registraron elevadas lecturas de magnesio $\left(4,7 \mathrm{~g} \mathrm{~L}^{-1}\right)$ y en otras de esta provincia como Baya y Diamante, y para Jujuy como Catal y Vilama (Fig. 1A; sitio 14), altas concentraciones de arsénico $\left(8,9 \mathrm{mg} \mathrm{L}^{-1}, 84 \mathrm{mg} \mathrm{L}^{-1}\right.$, $13 \mathrm{mg} \mathrm{L}^{-1}$ y $9 \mathrm{mg} \mathrm{L}^{-1}$, respectivamente).

Desde el punto de vista taxonómico, se identificaron 73 géneros con 375 taxones infragenéricos, muchos de ellos registrados por primera vez para el país (29) o para la región de estudio (331). La riqueza específica varió entre 7 y 69, en las lagunas Runtuyoc (Jujuy) y Lampacito (Catamarca, Fig. 1B; sitio 2), respectivamente. Se encontraron 168 taxones exclusivos de Catamarca, 70 de Jujuy y 130 especies fueron comunes a ambas provincias.

Los géneros mejor representados en cuanto al número de especies fueron Nitzschia Hassall (43), Navicula Bory (37), Pinnularia (Ehrenberg) Cleve (21), Surirella Turpin (17), Planothidium Round and Bukhtiyarova (15), Halamphora Lepkov (13), Craticula Grunow (12), Amphora Ehrenberg ex Kützing (11) y Stauroneis Ehrenberg (10).

No se encontraron especies que estuvieran 


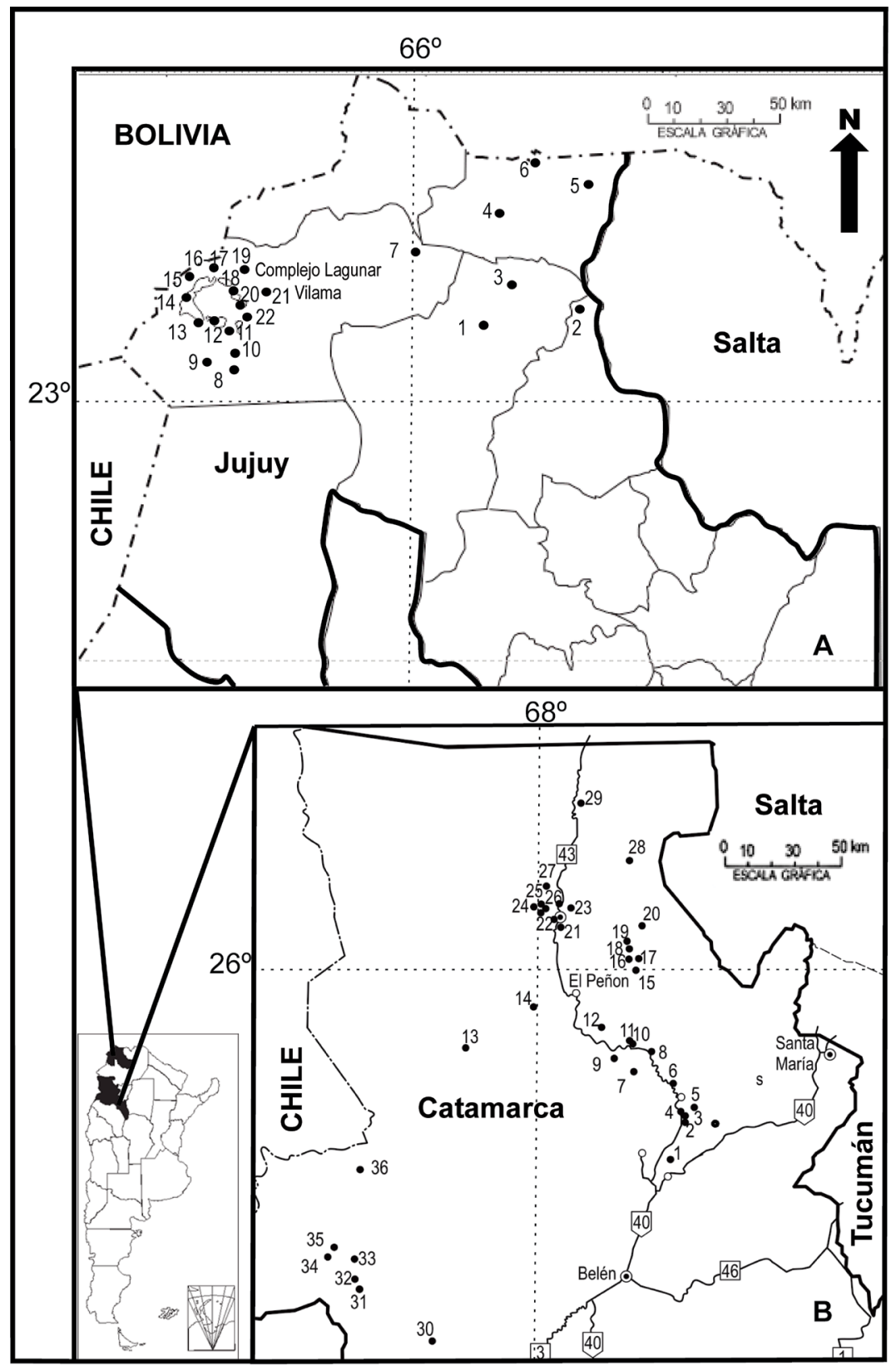

Fig. 1. Mapa del área de estudio. A: Ubicación de los sitios de muestreo en la provincia de Jujuy. B: Ubicación de los sitios de muestreo en la provincia de Catamarca.

presentes en los 57 humedales estudiados. Cuarenta y ocho taxones infraespecíficos (12,8\%) tuvieron una frecuencia de ocurrencia mayor al $18 \%$ (encontrados en más de 10 humedales) y 10 taxones $(2,7 \%)$, una frecuencia de ocurrencia superior al $40 \%$ (encontrados en más de 24 humedales): Cocconeis placentula var. euglypta, Denticula elegans, Halamphora veneta, Navicula lauca, $N$. parinacota, $N$. salinicola var. salinicola, $N$. veneta, Nitzschia bergii, N. halloyi y Tryblionella hungarica. 


\section{T. Seeligmann y N. I. Maidana - Altitud y salinidad vs. diatomeas de gran altitud}

El mayor número de especies (69) se registró en la laguna oligohalina Lampacito, con una salinidad inferior a $4,75 \mathrm{~g} \mathrm{~L}^{-1} \mathrm{y}$ estos valores se reducen a 11 especies en las lagunas hiperhalinas La Lagunita y Guinda y a 7 especies en la laguna de agua dulce Runtuyoc. Dentro de los máximos registros de salinidad (99 $\mathrm{g} \mathrm{L}^{-1}$ y $\left.104 \mathrm{~g} \mathrm{~L}^{-1}\right)$, la riqueza específica no fue mayor a 16. La figura 3 releja la escasa relación encontrada entre la riqueza específica y la salinidad en los humedales estudiados.

Las 10 especies, antes mencionadas, que tuvieron una frecuencia de ocurrencia mayor al 40 $\%$ se encontraron en aguas oligohalinas $\left(0,5-5 \mathrm{~g} \mathrm{~L}^{-1}\right)$ (Fig. 4), mientras que en aguas dulces $\left(<0,5 \mathrm{~g} \mathrm{~L}^{-1}\right)$ y polihalinas $\left(18-30 \mathrm{~g} \mathrm{~L}^{-1}\right)$ se observó una ausencia importante de algunos de estos taxones. En aguas dulces, los más frecuentes fueron Navicula lauca y Halamphora veneta y en las polihalinas, no se destacó ninguna especie por su frecuencia de ocurrencia. También se observó que Navicula parinacota y Nitzschia bergii parecen preferir aguas con salinidades más elevadas.

En cuanto a la relación entre la altura $(\mathrm{m} \mathrm{snm})$ y el número de especies, (Fig. 5), se observa que la riqueza fue similar en un amplio rango altitudinal.

Entre los 3800 y $4100 \mathrm{~m}$ snm se encontraron los 9 géneros más frecuentes (Fig. 6) y se destacaron en este nivel por el mayor número de especies Halamphora, Navicula, Nitzschia y Surirella. Entre los 4301 y los $4500 \mathrm{~m}$ snm ocurrió una importante disminución de la riqueza y solo Navicula y Nitzschia estuvieron bien representadas. Pinnularia y Stauroneis, que no se hallaron en este rango altitudinal, reaparecieron a partir de los $4500 \mathrm{~m}$ snm. En este último nivel de elevaciones, Nitzschia y Navicula fueron los géneros mejor representados, con 22 y 18 especies respectivamente, Planothidium y Surirella con 7, Pinnularia con 6 y Amphora con 5.

Especies como Amphora affinis, Navicula cryptotenelloides, $N$. perminuta y Craticula buderi, que si bien han sido halladas en sitios no montañosos de otras partes del país, se registraron solo en muestras ubicadas a partir de los $3500 \mathrm{~m}$ snm.

Más del $43 \%$ de taxones fueron encontrados en una o dos muestras, en cuerpos de agua dulce como laguna Morada (Jujuy, Fig. 1A; sitio 2), mesohalinos como la vega de la laguna Negra (Catamarca, Fig. 1B; sitio 34) y en hiperhalinos como las lagunas Diamante y Vilama. En relación a la altitud, de los 127 taxones registrados en un solo humedal, el $84 \%$ estuvo en humedales ubicados por arriba de los $4000 \mathrm{~m} \mathrm{snm}$.

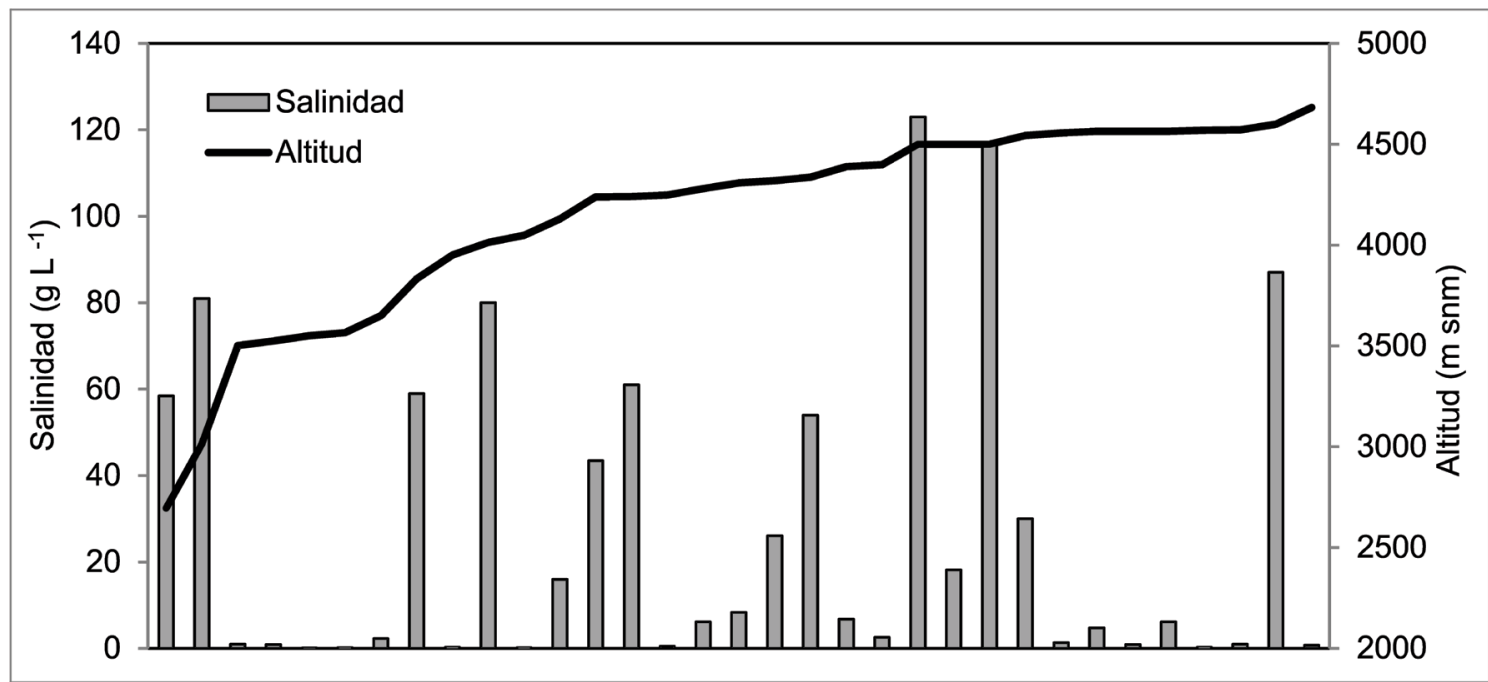

Fig. 2. Relación entre las variables altitud (m snm) y salinidad $\left(g^{-1}\right)$. 
Bol. Soc. Argent. Bot. 54 (4) 2019

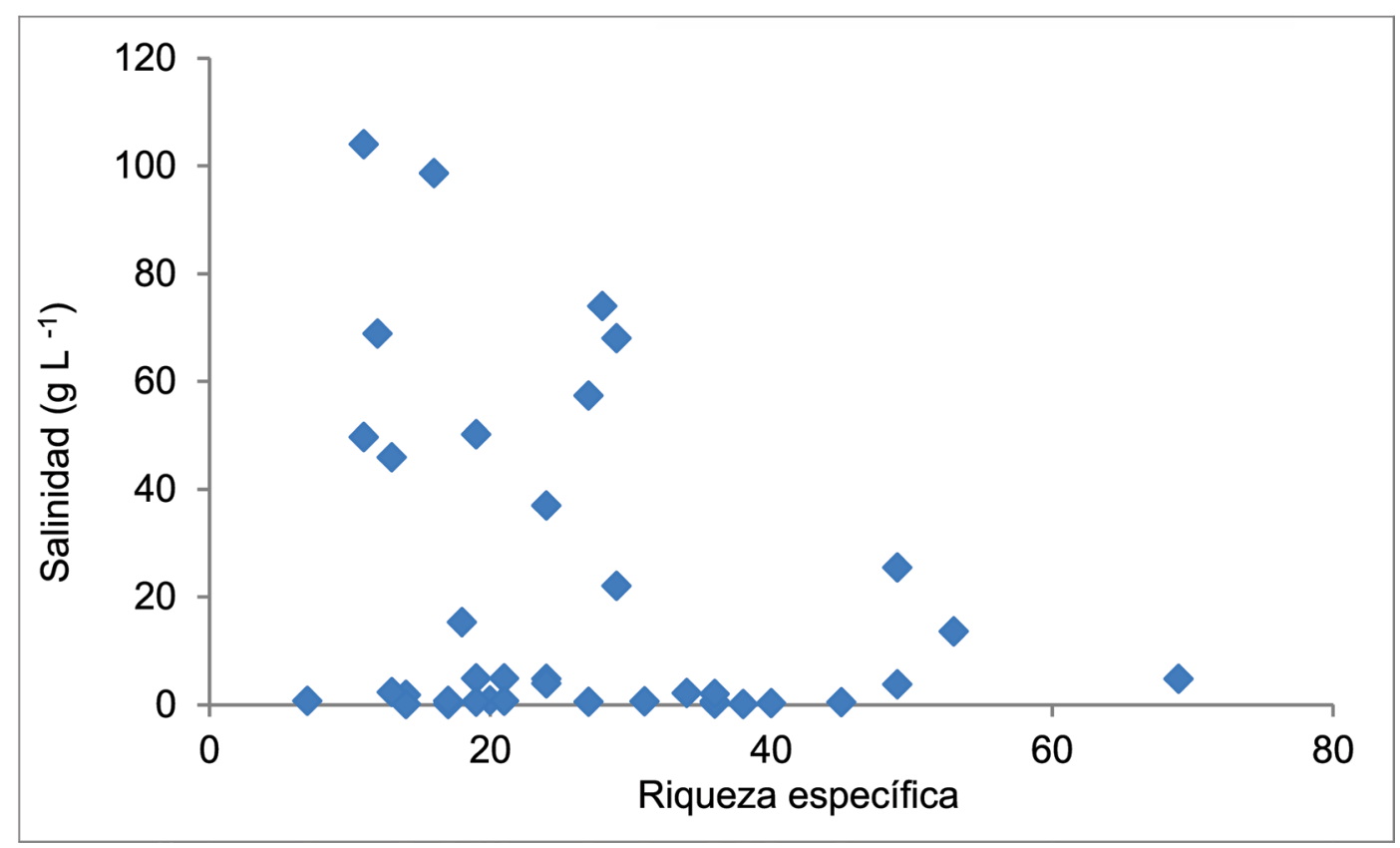

Fig. 3. Relación entre las variables riqueza específica y salinidad.

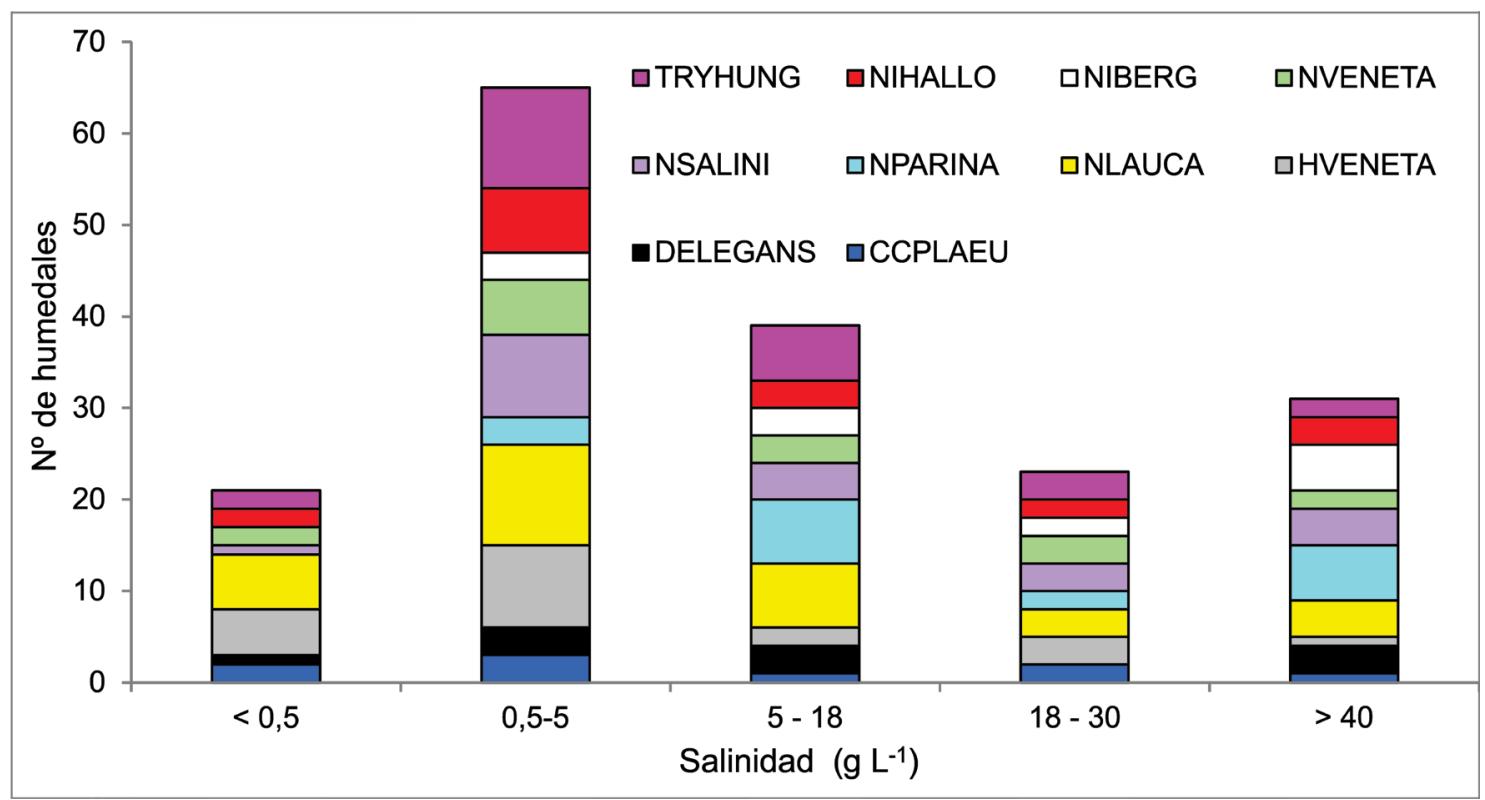

Fig. 4. Distribución de las especies Cocconeis placentula var. euglypta (CCPLAEU), Denticula elegans (DELEGANS), Halamphora veneta (HVENETA), Navicula lauca (NLAUCA), N. parinacota (NPARINA), N. salinicola (NSALINI), N. veneta (NVENETA), Nitzschia bergii (NIBERG), N. halloyi (NIHALLO) y Tryblionella hungarica (TIHUNG) en relación a gradientes de salinidad. 
C. T. Seeligmann y N. I. Maidana - Altitud y salinidad vs. diatomeas de gran altitud

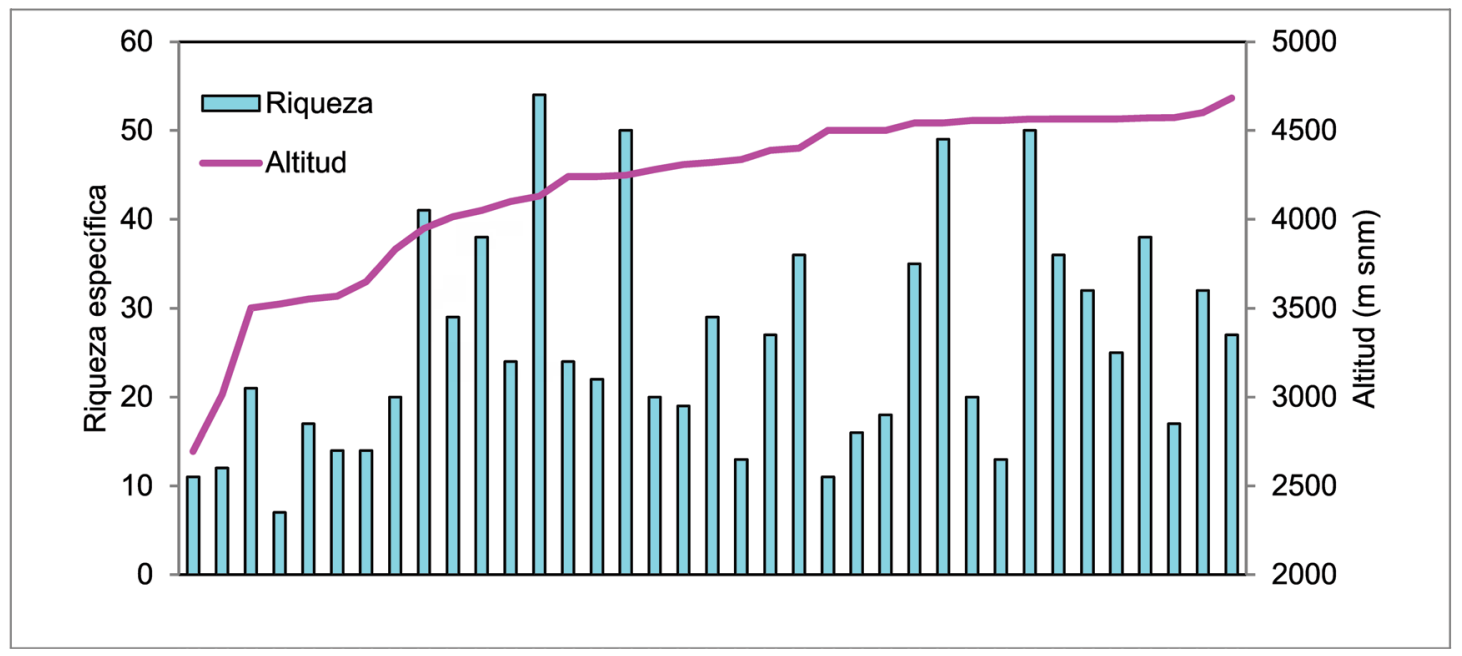

Fig. 5. Relación entre riqueza específica y altura.

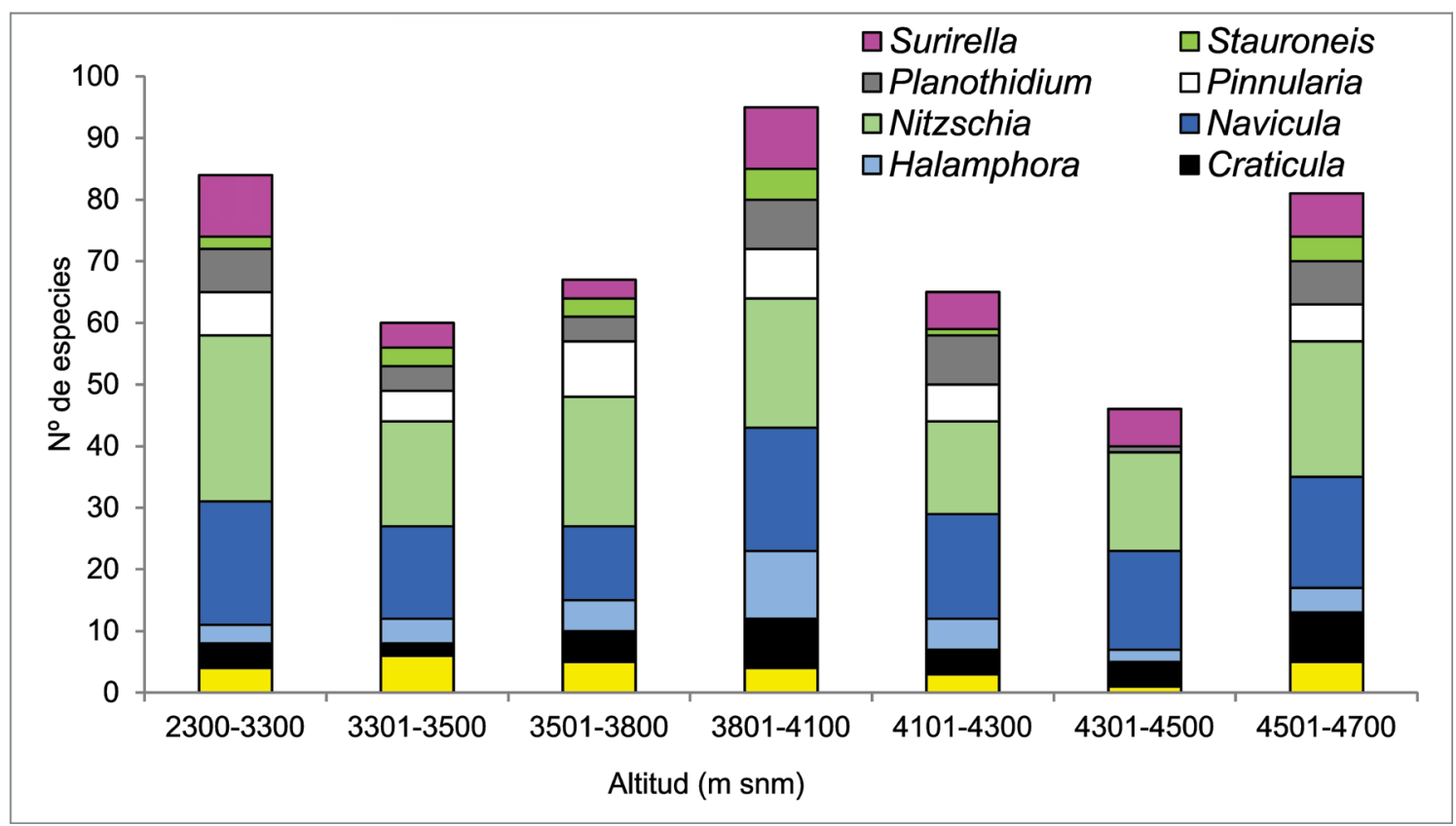

Fig. 6. Distribución de los géneros más frecuentes en relación a gradientes altitudinales.

\section{Discusión y Conclusiones}

La mayor parte de los humedales de alta montaña son salinos debido a la tasa diferencial entre precipitación y evaporación, lo que determina una acumulación de sales (Hammer, 1986) y la concentración salina se ve favorecida por la presencia de cuencas arreicas en zonas con climas áridos (Williams et al., 1990).

De acuerdo a Blinn (1993) la predominancia de los aniones cloruros y sulfatos se relaciona con la latitud y los amplios rangos de proporciones de iones 
dependen, en gran parte, de su capacidad diferencial de precipitación. Bajo condiciones de evaporación altas, precipita primero el $\mathrm{CaCO}_{3}$ permitiendo que los iones $\mathrm{SO}_{4}=$ y $\mathrm{Cl}^{-}$se concentren en el agua. Nauwerck (1994) encontró, en humedales de alta montaña de Suiza, que a mayor altitud ocurría un incremento relativo de los sulfatos y cloruros.

La Puna y la Prepuna se caracterizan, en general, por su homogeneidad climática lo que marca una diferencia notable entre los sitios investigados con los estudiados por Blinn (1993). En el Complejo Lagunar Vilama, una de las zonas de la Puna jujeña analizadas en esta contribución, la altitud varía solo algunos metros entre un cuerpo de agua y otro $\mathrm{y}$, sin embargo, hay diferencias importantes en la concentración y composición iónica entre cada uno de los humedales de este complejo (Salusso et al. 1997; Maidana et al. 2009). Así, el cloruro de sodio predomina en las lagunas Vilama, Palar, Colpayoc y Catal y el sulfato de sodio lo hace en Arenal, Pululos, Caití, Isla Grande y Cerro Negro. El factor dominante de influencia sobre los tipos de agua analizados estaría determinado más por el aporte del sustrato rocoso que por el clima local (Alonso, 2006).

El promedio de conductividad registrado en los humedales salinos de la Región Andina fue de 47,9 $\mathrm{mS} \mathrm{cm}{ }^{-1}$, muy similar al de los ambientes marinos $\left(48 \mathrm{mS} \mathrm{cm}^{-1}\right)$, en la mayoría de ellos se destacó el cloruro de sodio y muy pocos tuvieron sulfatos o bicarbonatos como dominantes. Los elevados registros de magnesio y/o sulfatos de algunos salares de la Puna, tales como los encontrados en las lagunas Baya, Caiti y Carachi Pampa, son el producto de una intensa actividad volcánica durante el Mioceno que se extendió hasta el Cuaternario en el sur de la Puna (Alonso, 2006; Risacher \& Ritz, 1991).

No encontramos una relación clara entre la conductividad y los tipos de iones dominantes ya que cuando hubo dominancia de $\mathrm{MgSO}_{4}$ y/o $\mathrm{NaCl}$ se registraron bajos y altos valores de conductividad.

Los primeros bioensayos en los que se vinculó la distribución de especies de diatomeas con diferentes gradientes de salinidad fueron realizados en su mayor parte en relación a la variación de una única sal, el cloruro de sodio (Herbst \& Blinn, 1998). Hecky \& Kilham (1973) afirman que la química del agua tiene una influencia significativa sobre las diatomeas. Blinn (1993) encontró que el número de especies fue, en promedio, mayor en ambientes dominados por cloruro de sodio que en aquellos donde predominaban los sulfatos y que cuando las concentraciones de $\mathrm{MgSO}_{4}$ eran medias $\left(\mathrm{Mg}^{+2}=44,8\right.$ meq $\mathrm{L}^{-1}$ y SO $_{4}^{-2}=103 \mathrm{meq} \mathrm{L}^{-1}$ ) se verificaba una alta riqueza específica de diatomeas mientras que por arriba o por debajo de estos valores el número de especies era drásticamente menor.

La importancia de la composición iónica sobre la comunidad de diatomeas se hizo evidente cuando otros autores (Potapova \& Charles, 2003) incluyeron en sus estudios distintos tipos de sales y observaron que la composición diatomológica estuvo fuertemente correlacionada con la proporción de cationes monovalentes/bivalentes.

En este trabajo, los humedales con elevados contenidos de magnesio y/o sulfatos, en general, mostraron menores valores de riqueza específica, en coincidencia con lo mencionado por Blinn (1993). En particular, en las lagunas Baya y Carachi Pampa, con altas conductividades $\left(54 \mathrm{~g} \mathrm{~L}^{-1}\right.$ y $84 \mathrm{~g} \mathrm{~L}^{-1}$ respectivamente) y con sales sulfatadas fue donde la riqueza no superó las 13 especies. Estos humedales tuvieron en común la presencia de Discostella stelligera, Nitzschia epithemoides y Surirella wetzeli. Las dos últimas especies son frecuentes en ambientes salinos pero la primera es una especie oligohalobiahalofila por lo que su presencia podría explicarse por transporte desde cuerpos de agua con menor salinidad.

Herbst \& Blinn (1998) hallaron, también en sus estudios experimentales, que existía una drástica disminución de la riqueza específica cuando la salinidad era superior a $50 \mathrm{~g} \mathrm{~L}^{-1}$ como consecuencia de la reducción de la actividad fotosintética. En los humedales estudiados con salinidad superior a $37 \mathrm{~g} \mathrm{~L}^{-1}$, se registraron entre 11 y 29 especies, similar a lo encontrado para valores de salinidad entre $0,7 \mathrm{~g} \mathrm{~L}^{-1}-25,4 \mathrm{~g} \mathrm{~L}^{-1}$. A distintas salinidades, la riqueza específica fue muy variable: en humedales oligohalinos fue entre 7-69, en los mesohalinos entre 19-54 y en los polihalinos entre 29-49. Estos resultados muestran que el número de especies encontradas no tuvo una clara relación con la salinidad.

En relación a la distribución de los taxones y el grado de salinidad de los humedales en los que se las encontró, se observaron tres situaciones: a) especies con amplia distribución y tolerancia a los distintos tenores de salinidad como Cocconeis euglypta, Denticula elegans, Halamphora veneta, 


\section{T. Seeligmann y N. I. Maidana - Altitud y salinidad vs. diatomeas de gran altitud}

Luticola mollis, Navicula cryptotenella, N. lauca, $N$ libonensis, $N$. pseudogracilis, $N$. salinicola var. salinicola, $N$. veneta, Nitzschia halloyi, Tryblionella hungarica y Surirella utahensis. b) especies que fueron encontradas en pocos humedales y que igualmente mostraron amplia tolerancia a diferentes salinidades (desde dulces a hiperhalinas) como Luticola cohni, Navicula atacamana y Nitzschia gracilis y c) aquellas especies que si bien estuvieron presentes en numerosos humedales, solo lo fueron a partir de aguas oligohalinas como Nitzschia epithemoides, N. pusilla y Surirella wetzelii.

En ambientes montañosos, el incremento en altura va acompañado de una disminución de la temperatura y un aumento de la radiación UV, dos factores que afectan la actividad fotosintética disminuyendo, en consecuencia, el número de aquellas especies capaces de adaptarse a estas condiciones (Lukavsky, 1994; Sommaruga, 2001; Álvarez Blanco et al., 2011; Frau et al., 2015, entre otros) además de la reducida disponibilidad de recursos (Rautio, 2001). En nuestros estudios registramos, por un lado, una alta variabilidad en composición específica de las diatomeas en humedales ubicados a altitudes similares, como es el caso del Complejo Lagunar Vilama y, por otro, encontramos que, en el rango altitudinal entre 2300 y $3300 \mathrm{~m}$ snm, la riqueza fue menor (133 especies) que a mayor altitud (4500$4700 \mathrm{msnm}$ ) donde registramos 167 especies. Todo lo expuesto nos lleva a concluir que la altura per se y las condiciones ambientales que esta genera no serían las variables condicionantes de la riqueza y/o composición de diatomeas en este tipo de ambientes.

En el gradiente altitudinal analizado, la comunidad de diatomeas estuvo formada principalmente por especies de los géneros Navicula y Nitzschia, especies típicamente bentónicas cuya presencia en las muestras de agua libre se podría atribuir a las altas tasas de turbulencia de estos sistemas (Alvial et al. 2008; Frau et al., 2015). Si bien la altitud y salinidad interactúan y son importantes estresores determinantes de la riqueza específica como lo señalan Frau et al. (2015), existen otros factores que afectan el número de especies, tales como como la permanencia del cuerpo de agua, la composición química, la irradiación, los vientos, la herbivoría, etc. En este sentido, los cambios que pueden ocurrir en la estructura de la comunidad fitoplanctónica están probablemente asociados con alta tasas de turbulencia (Blanco et al., 2013).
La ausencia de especies comunes, aún entre aquellos humedales ubicados próximamente entre sí, como en el caso del Complejo Lagunar Vilama señala la importante variabilidad existente entre ellos. El alto porcentaje de taxones hallados una sola vez a partir de los $4000 \mathrm{~m}$ snm y la presencia de especies reportadas por primera vez para ambientes de altura, sugieren intensificar estudios que revelen la posible existencia de alguna dimensión del nicho ecológico no detectada hasta el momento.

En relación a la distribución y registro de especies, muy pocas $(1,6 \%)$, y todas ellas consideradas cosmopolitas, aparecieron exclusivamente por debajo de los $3000 \mathrm{~m}$ snm. Entre los taxones que parecen preferir vivir a mayor altitud, 35 fueron hallados solo en humedales ubicados por encima de los $4200 \mathrm{~m}$ snm (Achnanthes coarctata, Anomoeoneis aff. var. angusta morf. 2, A. sp, Caloneis schumanniana, Chaetoceros muelleri, Cocconeis neuquina, Discostella stelligera, Cymbella subcistula, Diploneis chilensis, Eunotia incisa, Fallacia monoculata, Frustulia crassinervia, F. saxonica, Gomphonema fereformosum, Gyrosigma balticum, Halamphora coffaeformis, Halamphora sp, Navicula phylleptosoma, Naviculadicta sp, Neidium affine, N. bisulcatum var. subundulatum, Nitzschia amphibia fa. umbrosa, $N$. compresa, $N$. elegantula, N. schwabei, N. sigma, P. perincognita, Planothidium elipticum, P. pericavum, P. quadripunctatum, Rhopalodia brebisonii, Stauroneiss valbardensis, S.latistauros, Staurophora elata y Thalasiosira patagónica). Entre estas especies, destacamos a Pulchella schwabei (Krasske) Krammer que fue hasta el momento encontrada sólo en Sudamérica; a Cocconeis neuquina, Diploneis chilensis, Nitzschia amphibia fa. umbrosa, Stauroneis latistauros, S. svalbardensis y Spicaticribra patagónica (Maidana) Kociolek \& Khursevich, como exclusivamente americanas.

Esta contribución, que abarcó numerosos registros de una región pobremente conocida en cuanto a la diversidad de microalgas, nos permitió relacionar los niveles de salinidad y altura para algunas especies de diatomeas, con lo que podemos ampliar sus rangos de tolerancia hasta ahora conocidos para estos taxones, condiciones no reportadas previamente en la bibliografía. Ejemplos de esta situación serían Amphora affinis, Craticula buderi, Navicula cryptotenella y $N$. perminuta que no habían sido registradas hasta el momento en ambientes de altura. 
Por su parte, Navicula lauca, junto a $N$. parinacota y $N$. peregrina, fueron encontradas en humedales hiperhalinos (Maidana et al., 2011), a diferencia de lo esperado a partir de la información dada por Lange-Bertalot (2001), que las menciona para ambientes con bajos valores de salinidad. Otros taxones, como Anomoeoneis sphaerophora fa. costata, Craticula halophila, Gomphonema parvulum, Halamphora veneta, Navicula cryptocephala, N. cryptotenella, Nitzschia frustulum, N. gracilis, N. palea, Pinnularia microstauron, Planothidium delicatulum y Surirella brebissoni fueron encontradas en aguas con salinidades muy superiores a las indicadas como óptimas por Martínez Macchiavello \& Salas Aramburu (1994), Wilson et al. (1996), Potapova \& Charles (2003) y Licursi et al. (2010).

Además, Anomoeoneis sphaerophora fa. costata, Denticula elegans, Diploneis chilensis, Navicula lauca, $N$. parinacota, $N$. peregrina, $N$. gracilis, $N$. liebetruthii, N. pusilla, Surirella brebissonii y $S$. utahensis parecen ser tolerantes a salinidades muy altas, ya que fueron encontradas en lagunas con el máximo registro $\left(105 \mathrm{~g} \mathrm{~L}^{-1}\right)$.

De los resultados obtenidos, se desprende la relevante valoración ecológica de la comunidad de diatomeas como descriptora ambiental, remarcando la necesidad de incorporar nuevos relevamientos en áreas montañosas para encontrar respuestas a estos y otros interrogantes todavía no resueltos.

\section{Contribución de los autores}

CTS y NIM diseñaron y realizaron la investigación. CTS coleccionó el material de campo. Ambas autoras participaron en la escritura del manuscrito.

\section{Agradecimientos}

Deseamos agradecer a la Fundación Miguel Lillo por el lugar de trabajo proporcionado.

\section{Bibliografía}

ALONSO, R. 2006. Ambientes Evaporíticos Continentales de Argentina. INSUGEO, Ser. Correl. Geol. 21: 155-170.
ÁlVAREZ-BLANCO, I., C. CEJUDO-FIGUEIRAS, I. DE GODOS, R. MUÑOZ \& S. BLANCO. 2011. Las diatomeas de los salares del Altiplano boliviano: singularidades florísticas. Bol. R. Soc. Esp. Hist. Nat. Sec. Biol. 105: 67-82.

ALVIAL, I. E., F. J. CRUCES, A. E. ARANEDA, M. GROSJEAN \& R. E. URRUTIA. 2008. Estructura comunitaria de diatomeas presentes en los sedimentos superficiales de ocho lagos andinos de Chile central. Revista Chilena de Historia Natural 81: 83-94. https://doi.org/10.4067/S0716-078X2008000100007

BLANCO, S., I. ÁLVAREZ-BLANCO, C. CEJUDOFIGUEIRAS, I. DE GODOS, E. BÉCARES, R. MUÑOZ, H. O. GUZMAN, V. A. VARGAS \& R. SOTO. 2013. New diatom taxa from high-altitude Andean saline lakes. Diatom Res. 28: 13-27. https://doi.org/10.1080/0269249X.2012.734528

BLINN, D.W. 1993. Diatom community structure along physicochemical gradients in saline lakes. Ecology 74: 1246-1263. https://doi.org/10.2307/1940494

BUCHER, E.H. 2006. Bañados del Río Dulce y Laguna de Mar Chiquita. Córdoba-Argentina. Academia Nacional de Ciencias (Córdoba, Argentina).

CAZIANI, S. \& E. DERLINDATI. 2000. Abundance and habitat of Andes flamingos in northwestern Argentina. Waterbirds 23: 121-133.

https://doi.org/10.2307/1522157

CAZIANI, S., E. DERLINDATI, A. TALAMO, A. SUREDA, C.E. TRUCCO \& G. NICOLOSSI. 2001. Waterbird richness in altiplano wetlands of Northwestern Argentina. Waterbirds 24: 103-117. https://doi.org/10.2307/1522249

COWARDIN, L. M., V. CARTER, F.C. GOLET \& E.T. LAROE. 1979. Classification of. wetlands and deepwater habitats of the United States. U. S. Department of the Interior, Fish and Wildlife Service, Washington, D.C.

DEJOUX, C. 1993. Benthic invertebrates of some lakes of SudLipez region, Bolivia. Hydrobiologia 267: 257-267.

https://doi.org/10.1007/978-94-011-2076-0_21

DE WOLF, H. 1982. Method of coding of ecological data from diatoms for computer utilization. Mededel. Rijks Geol. Dienst. 36: 95-110.

DÍAZ, C. \& N. I. MAIDANA. 2005. Diatomeas de los salares de Atacama y Punta Negra II región. Chile. Centro de Ecología Aplicada, Santiago.

FRAU, D., Y. BATTAUZ., G. MAYORA \& P. MARCONI. 2015. Controlling factors in planktonic communities 


\section{T. Seeligmann y N. I. Maidana - Altitud y salinidad vs. diatomeas de gran altitud}

over a salinity gradient in high-altitude lakes. Ann. Limnol. - Int. J. Lim. 51: 261-272. https://doi.org/10.1051/limn/2015020

GARCÍA FERNÁNDEZ, J. \& Y. R. TECCHI. 1991. La Reserva de la Biósfera Laguna de los Pozuelos: un ecosistema pastoril en los Andes centrales. PERINBIAL UNJu, Argentina, Unesco ORCYTMAB.

GONZÁlEZ ACHEM, A. L., C. T. SEELIGMANN \& M. ALDERETE. 2014. Variaciones espaciotemporales de la flora diatomológica en Laguna de Los Pozuelos (Jujuy, Argentina). Bol. Soc. Argent. Bot. 49: 177-193.

HAMMER, U. T. 1986. Saline Lake Ecosystems of the World, Junk Publishers, Dordrecht, Holland.

HECKY, R. \& P. KILHAM. 1973. Diatoms in alkaline, saline lakes: ecology and geochemical implications. Limnol. Oceanogr. 18: 53-71. https://doi.org/10.4319/1o.1973.18.1.0053

HERBST, D. \& D. BLINN. 1998. Experimental mesocosm studies of salinity effects on the benthic algal community of saline lake. J. Phycol. 34: 772-778. https://doi.org/10.1046/j.1529-8817.1998.340772.x

LANGE-BERTALOT, H. 2001. Diatoms of Europe 2. Navicula sensu stricto. 10 Genera Separated from Navicula sensu lato. Frustulia. A.R.G. Gantner Verlag, Ruggell.

LICURSI, M., N. GÓMEZ \& J. DONADELLI. 2010. Ecological optima and tolerances of coastal bethic diatoms in the freshwater-mixohaline zone of the Río de la Plata estuary. Mar. Ecol. Prog. Ser. 418: 105-117. https://doi.org/10.3354/meps08865

LOWE, R. L. 1974. Environmental requirements and pollution tolerance of freshwater diatoms. National Environmental Research Center. U. S. Environmental Protection Agency, Cincinnati, Ohio.

LUKAVSKY, J. 1994. Algal flora of the High Tatra Mountains (Slovakia). Hydrobiologia 274: 65-74. https://doi.org/10.1007/978-94-017-2095-3_8

MAIDANA, N.I., M.S. VIGNA \& V. MASCITTI.1998. Ficoflora de la Laguna de Pozuelos (Jujuy, Argentina) I: Bacillariophyceae. Bol. Soc. Argent. Bot. 33: 171-179.

MAIDANA, N.I. \& C.T. SEELIGMANN. 2006. Diatomeas (Bacillariophyceae) de ambientes acuáticos de altura de la Provincia de Catamarca, Argentina II. Bol. Soc. Argent. Bot. 41: 1 - 13.

MAIDANA, N.I. \& C.T. SEELIGMANN. 2015. Diatomeas (Bacillariophyceae) en humedales de altura de la provincia de Catamarca (Argentina). III. Bol. Soc. Argent. Bot. 50: 447-466.
MAIDANA, N.I., C.T SEELIGMANN \& M. R. MORALES. 2009. Bacillariophyceae del Complejo Lagunar Vilama (Jujuy, Argentina). Bol. Soc. Argent. Bot. 44: 257-271.

MAIDANA, N.I., C.T. SEELIGMANN \& M.R. MORALES. 2011. El género Navicula sensu stricto (Bacillariophyceae) en humedales de altura de Jujuy, Argentina. Bol. Soc. Argent. Bot. 46: 13-29.

MARTÍNEZ MACCHIAVELLO, J.C. \& W. SALAS ARANBURU. 1994. Catálogo taxonómico ecológico de las diatomeas no-marinas recientes y fósiles de la República Argentina. Instituto Antártico Argentino. Buenos Aires.

MASCITTI, V. 1996. Partición del recurso en la comunidad de flamencos de Laguna Los Pozuelos, Jujuy, Argentina. Tesis Doctoral N ${ }^{\circ} 2904$ UBA.

MORALES, E.A., M.H. NOVAIS, G. CHÁVEZ, L. HOFFMANN, \& L. ECTOR. 2012. Diatoms (Bacillariophyceae) from the Bolivian Altiplano: three new araphid species from the Desagüadero River draining Lake Titicaca. Fottea 12: 41-58. https://doi.org/10.5507/fot.2012.004

MORRONE, J. J. 2006. Biogeographic areas and transition zones of Latin America and the Caribbean islands based on panbiogeographic and cladistics analyses of the entomofauna. Annual Rev. Entomol. 51: 467-494.

https://doi.org/10.1146/annurev.ento.50.071803.130447

NAUWERCK, A. 1994. A survey on water chemestry and plankton in high mountain lakes in northen swedish lapland. Hydrobiologia 274: 91-100. https://doi.org/10.1007/978-94-017-2095-3_11

POTAPOVA, M. \& D. CHRALES. 2003. Distribution of benthic diatoms in U.S. rivers in relation to conductivity and ionic composition. Freshwater Biol. 48: 1311-1328. https://doi.org/10.1046/j.1365-2427.2003.01080.x

RAUTIO, M. 2001. Zooplankton assemblages related to environmental characteristics in treeline ponds in Finnish Lapland. Arct. Antarct. Alp. Res. 33: 289-298. https://doi.org/10.1080/15230430.2001.12003433

REJAS, D., C. VALVERDE, C.E. FERNÁNDEZ. 2012. Limitación por nutrientes y pastoreo como factores de control las densidades de bacterias y algas planctónicas en una laguna altoandina (Cochabamba, Bolivia). Rev. Bol. Ecol. y Cons. Amb. 30: 1-12.

RISACHER, F. \& B. RITZ. 1991. Geochemestry of Bolivian salars, Lipez, southern Altiplano: origin of solutes and brine evolution. Geochim. Cosmochim. Acta. 55: 687-705. https://doi.org/10.1016/0016-7037(91)90334-2 
RUMRICH, U., H. LANGE-BERTALOT \& M. RUMRICH. 2000. Diatoms of the Andes: from Venezuela to Patagonia/Tierra del Fuego. Iconogr. Diatomol. 9: 1-649.

SALUSSO, M., S. M. CAZIANI, \& S. M. MORAÑA. 1997. Distribución de plancton en relación a los caracteres limnológicos y a la avifauna de la Puna Jujeña. CAL II.

SEELIGMANN, C.T. \& N.I. MAIDANA. 2003. Diatomeas (Bacillariophyceae) en ambientes de altura de la provincia de Catamarca (Argentina). Bol. Soc. Argent. Bot. 38: 39-50.

SEELIGMANN, C.T. \& N.I. MAIDANA. 2013. ¿Existen especies de Navicula (Bacillariophyta) exclusivas de ambientes de alta montaña en Argentina? Bol. Soc. Argent. Bot. 48: 421-433.

SEELIGMANN, C.T., N.I. MAIDANA \& M. MORALES. 2008. Diatomeas (Bacillariophyceae) de humedales de altura de la Provincia de Jujuy - Argentina. Bol. Soc. Argent. Bot. 43: 1-517.

SERVANT-VILDARY, S. \& M. BLANCO. 1984. Les diatomées fluvio-lacustres plio-pléistocènes de la formation Charaña (Cordillère occidentale des Andes, Bolivie). Cahiers ORSTOM, Série Géologie 14: 55-102.

SOMMARUGA, R. 2001. The role of UV radiation in the ecology of alpine lakes. J. Photochem. Photobiol., B. 62: $35-42$. https://doi.org/10.1016/S1011-1344(01)00154-3

VAN DAM, H., A. MERTENS \& J. SINKELDAM. 1994. A coded checklist and ecological indicator values of freshwater diatoms from the Netherlands. Netherlands J. Aq. Ecol. 28: 117-133. https://doi.org/10.1007/BF02334251

WILLIAMS, W. D. 1993. Conservation of salt lakes. Hydrobiologia 267: 291-306. https://doi.org/10.1007/978-94-011-2076-0_23

WILSON, S.E., B.F. CUMMING \& J.P. SMOL. 1996. Assessing the reliability of salinity inference models from diatoms assemblages: an examination of 219 lake data set from western North America. Can. J. Fish. Aquat. Sci. 53: 1580-1594. https://doi.org/10.1139/cjfas-53-7-1580 